\title{
Some Aspects of Processing and Properties of Composite Material with Si-C Particles
}

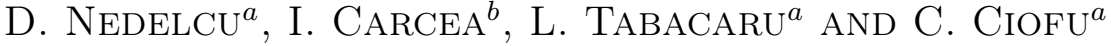 \\ ${ }^{a}$ Faculty of Machine Manufacturing and Industrial Management; \\ ${ }^{b}$ Faculty of Science and Materials Engineeringl "Gheorghe Asachi" \\ Technical University of Iasi, 700050 Iasi, Romania
}

\begin{abstract}
The processing of composite materials with metal matrix reinforced with carbon particles, graphite or $\mathrm{SiC}$ (Silicon Carbide) is decisively determined by the transfer of the reinforcing particles from the gaseous atmosphere into the molten metal and then by their transfer from liquid into solid. The degree of incorporation, the uniformity of particle distribution and finally all the properties of the composite materials depend on the way in which the transfer conditions from one phase to another have been accomplished. According to the thermodynamic principles, the transfer of the reinforcing particles from one phase to another can happen without external energy input only if the variation of the total free enthalpy is negative. The estimation of the direction of transfer can be done after the determination of critical measurements to the balance of forces that act on the reinforcing element. The particles wetted by the liquid metal and with density of its nearest will be integrated in the melt without external energy input, and those dry and light need additional energy consumption (powder injection, melt stirring etc.). The stalling speed of the $\mathrm{SiC}$ particles to the gas/liquid interface is influenced by the density difference and the reinforcing particles size. The critical moving speed of the solidification front is decisively influenced by the difference between $\sigma_{\mathrm{PS}}$ and $\sigma_{\mathrm{PS}}$, inter-phase tensions, as well as by the reinforcing particles size, if their density significantly differs from the one of the liquid alloy. Compared with the initially used alloy, the pull strength is doubled for some samples of obtained composite material. The ultrasonic testing disclosed the presence of some very small spots (porosities) which don't have a major influence on the tensile specimens subjected to pulling. From this reason, a very clear display of the bottom echo can be observed, which justifies the uniformity of the casting process. At the level of the matrix, the presence of the carbon to the separation limit between matrix and reinforcement can be observed, which confirms that during the processing Aluminium Carbide $\mathrm{Al}_{4} \mathrm{C}_{3}$ has been formed. The presence of these carbides considerably improves the wetting process.
\end{abstract}

PACS: 81.05.Mh, 81.05.Ni, 81.05.Pj, 81.05.Qk, 12.60.Rc

\section{The gas/liquid transfer of the reinforcing particles}

The transfer of a particle from gas to solid is complete when the particle enters the liquid up to the total replacement of the solid/gas interface with an equal solid/liquid interface.

Developing the thermodynamic principles proposed by Neuman et al., P.K. Rohatgi's [1] and Asthana P.'s modelling elements, or the simplified mechanical model launched by S. Kacar, et al. [2], B. Toshev [3], we realised a new model starting from the following assumptions: during all stages of sinking, the thermodynamic equilibrium of the surface to the phase junction is applied: the specific interfacial energy is isotropic, gas - liquid metal interface is plain and free of oxides, the surrounding reinforcing particles don't interact with each other, the effects of thermal gradients, the melt convection and the chemical reactions to the liquid metal - particle interface are negligible to full immersion, the reinforcing particle movement has been assimilated to the vertical plane-parallel motion on the distance $2 r$ of a rigid sphere with $r$ radius, as shown in Fig. 1.

Against one rigid sphere with $\mathrm{r}_{p}$ radius, $\mathrm{m}_{p}$ weight and $\rho_{p}$ density act the following forces [4]: $-\boldsymbol{G}$ force due to its weight

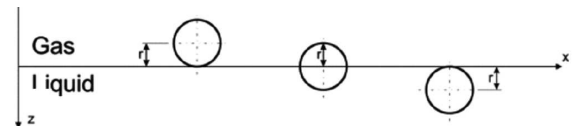

Fig. 1. The movement from gas to liquid on the $2 r$ distance of a rigid sphere with $r$ radius.

$$
\begin{gathered}
\boldsymbol{G}=m_{p} \boldsymbol{g}=4 \pi r_{p}^{3} \rho_{p} \boldsymbol{g} / 3, \\
-\boldsymbol{F}_{A} \text { buoyancy force } \\
F_{A}=\Delta V \rho_{L} G,
\end{gathered}
$$

the force due to the inter-phase tension, $F_{\sigma}$, in which: $\rho_{L}$ is the density of the liquid metal; $\Delta V$ is the volume of the dislodged metal by the rigid sphere at that moment; $\boldsymbol{g}$ is the gravitational acceleration.

$F_{A}$ and $F_{\sigma}$ forces can be calculated for some position when the centre of mass of the sphere has the $Z$ coordinate, which means that the sphere has vertically lowered on the $Z$ distance, as shown in Fig. 2.

$$
\begin{aligned}
& \text { We define } F_{\sigma} \text { as being: } \\
& F_{\sigma}=2 \pi a F_{1}=2 \pi a F \cos (\beta+\delta)
\end{aligned}
$$

The $F_{\sigma}$ force is the surface tension force per unit length of the contact contour, that is the surface tension $\sigma_{\mathrm{LG}}$. So:

$$
F_{\sigma}=2 \pi a \sigma_{\mathrm{LG}} \cos (\beta+\delta)
$$




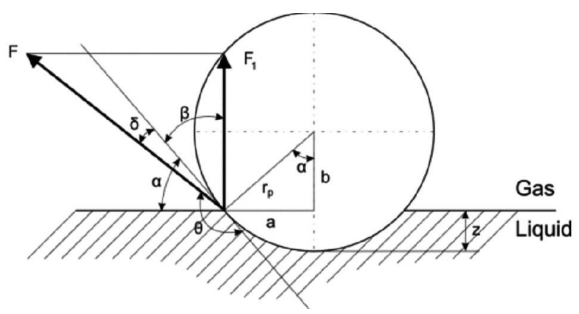

Fig. 2. Physical quantities in the particular complex system gas-liquid.

Because $a=r_{p} \sin \alpha$, and $\delta=\pi-\theta \operatorname{si} \beta=\pi / 2-\alpha$ follows:

$$
F_{\sigma}=2 \pi \sigma_{\mathrm{LG}} r_{p}\left(-\sin ^{2} \alpha \cos \theta-\sin \alpha \cos \alpha \sin \theta\right)
$$

or

$$
\begin{aligned}
& F_{\sigma}=2 \pi \sigma_{\mathrm{LG}}\left[-Z\left(2 r_{p}-Z\right) \cos \theta\right. \\
& \left.-\left(r_{p}-z\right) \sqrt{z\left(2 r_{p}-z\right)} \sin \theta\right] / r_{p} .
\end{aligned}
$$

We can observe that at the initial moment when $Z=0$, as well as after the complete immersion of the sphere into the liquid at $Z=2 r_{p}$, force $F_{\sigma}=0$.

The buoyancy force is:

$$
F_{A}=\pi z\left(3 a^{2}+z^{2}\right) \rho_{L} g / 3=\pi z^{2} \rho_{L} g\left(3 r_{p}-z\right) / 3(1.7)
$$

The resultant forces that act on the particle during the immersion are:

$$
\begin{gathered}
F=\pi g\left[4 r_{p}^{3} \rho_{p}-z^{2} \rho_{L}\left(3 r_{p}-z\right)\right] / 3 \\
-2 \pi \sigma_{\mathrm{LG}}\left[-z\left(2 r_{p}-z\right) \cos \phi\right. \\
\left.-\left(r_{p}-z\right) \sqrt{z\left(2 r_{p}-z\right)} \sin \phi\right] / r_{p} .
\end{gathered}
$$

Because this resultant force is conservative, the energy conservation law can be applied, and by their integration at $Z=2 r$ limit we can obtain the critical velocity expression of incorporation at the gas/liquid transfer of the reinforcing particles.

$$
V_{c r}=\left[-4 \sigma_{\mathrm{LG}} \cos \theta / r_{p} \rho_{p}-2 g r_{p}\left(2-\rho_{L} / \rho_{P}\right)\right]^{1 / 2} .
$$

As shown in the Eq. (1.9), the critical speed is determined by two different terms which are themselves influenced by the surface characteristics $\left(\sigma_{\mathrm{LG}} \mathrm{si} \theta\right)$, respectively by the difference between the density of the particle and of the liquid. The first term becomes positive and contributes to the critical speed increase when the contact angle $\theta$ is higher than $\pi / 2$. The greater $\theta$ value is comparing to $\pi / 2$, the greater critical speed is.

The second term becomes positive and contributes to the critical speed increase when the liquid density is at least twice the density of the particles.

In conclusion, the particles wetted by the liquid metal with the density close to its own density will be incorporated in the melt without external energy input, and those dry and light need additional energy consumption (powder injection, melt stirring etc).

\section{The liquid/solid transfer of the reinforcing particles}

The behaviour of the particles at the liquid/solid interface differs according to the way in which the solidification goes $[5,6]$. At the front plane solidification and unidirectional movement, the particles can be incorporated or expelled, and at the multidirectional solidification when the solidification front is divided into cells, dendrites or equal-axial granules, the particles can be incorporated, expelled or embedded $[7,8]$.

Thermodynamically speaking, the transfer through liquid/solid plane interfaces of the reinforcing particles is realised with a variation of the Helmholtz free energy of the system. If the variation of the total free energy $\Delta F_{t}$ is negative, then the particles are spontaneously incorporated, and if the $\Delta F_{t}$ variation is positive, then the particles will be expelled from the interface.

The kinetics of the reinforcing particles transfer from liquid to solid imposes the elucidation of the following problems: the nature of the rejection forces between liquid/solid interface and particles; the implied mechanism at the mass transfer; the influence of the particles on the shape of the interface.

The mathematical modelling of the solidification front plan relative movement against a reinforcing particle imposes the following restrictions: the reinforcing particle is spherical; the rejection force is given by the interfacial energy variation with the distance between the solidification front and particle; the balance corresponds to a distance between front and particle of two atomic diameters; the mass transfer is done only through convection; the different thermal conductivity determines the curving of the interface; the concentration gradient is negligible.

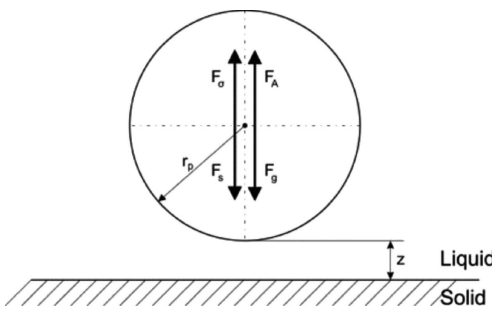

Fig. 3. The forces that act on a particle existing in the influence area of a solid/liquid plane, horizontal interface.

The forces that act against a spherical particle of $r_{p}$ radius and $\rho_{p}$ density that moves with $V_{p}$ speed in solidification front influence area, as shown in Fig. 3, are [9]: the gravitational force

$$
F_{g}=4 \pi r_{p}^{3} \rho_{p} g / 3
$$

the buoyancy force

$$
F_{A}=4 \pi r_{p}^{3} \rho_{L} g / 3
$$

the Stokes force

$$
F_{s}=6 \pi \eta r_{p} V_{p}
$$

the rejection force given by the interfacial energies 


$$
F_{\sigma}=2 \pi r \Delta \sigma
$$

in which

$$
\Delta \sigma=\Delta \sigma_{0}\left(d_{0} / z\right)^{n}, \quad \text { with } \quad n=2,3 \ldots 7
$$
and

$$
\Delta \sigma_{0}=\sigma_{P S}-\sigma_{\mathrm{PL}},
$$

where $\sigma_{\mathrm{PS}}$ is the particle-solid inter-phase tension and $\sigma_{P L}$ is the particle-liquid inter-phase tension.

The movement equation of the particles existing in front of a solidification front and horizontally is nonlinear and cannot be integrated but for particular cases, as at the equilibrium. In this case, we have:

$$
\begin{gathered}
V_{c r}=\left[\Delta \sigma_{0} d_{0} /(n-1) 2^{n-1}\right. \\
\left.\quad-4 r_{p}^{3} g\left(\rho_{p}-\rho_{L}\right) / 3\right] / 6 \eta r_{p} .
\end{gathered}
$$

The critical speed which must be touched by the solidification front in order to be able to incorporate the particle is given by the difference between two terms: the first term depends on the difference between interfacial tensions $\sigma_{\mathrm{PS}}$ and $\sigma_{\mathrm{PL}}$, by the inverse radius of the particle and inverse dynamic viscosity of the liquid; the second term depends on the difference between the densities of the particle and the liquid, on the square of the particle radius and on the inverse dynamic viscosity of the liquid.

It must be mentioned that the value of the second term is influenced by the angle made by the solidification front with the vertical of the place, as well as by the gravitational attraction value. If no gravitation implied, the second term is annulated.

\section{Experimental research on gas/liquid and liquid/solid transfer of the reinforcing particles}

In order to realise the research, aluminium alloys have been used, their chemical composition being given in Table I.
TABLE I

The chemical composition of the alloys used in experimentation.

\begin{tabular}{l|c|c|c|c|c|c}
\hline \hline \multirow{2}{*}{$\begin{array}{l}\text { Type of } \\
\text { alloy }\end{array}$} & \multicolumn{6}{|c}{ Chemical composition, [mass\%] } \\
\cline { 2 - 7 } & $\mathrm{Mg}$ & $\mathrm{Cu}$ & $\mathrm{Si}$ & $\mathrm{Fe}$ & $\mathrm{Mn}$ & $\mathrm{Al}$ \\
\hline ENACAlSi5Cu3Mg & 0.22 & 3.46 & 4.80 & 0.40 & 0.24 & rest \\
ENACAlSi7Cu2Mg & 0.59 & 2.31 & 7.30 & 0.41 & 0.22 & rest \\
AlMgSi0.5 & 0.49 & 0.04 & 0.33 & 0.20 & 0.05 & rest
\end{tabular}

For experimentation, particles of Silicon Carbide, SiC, with $3200 \mathrm{~kg} / \mathrm{m}^{3}$ toughness have been used. The critical speed of projection of some $\mathrm{SiC}$ particles with $20 \mu \mathrm{m}$ and $60 \mu \mathrm{m}$ granulation on gas/aluminium heated up to $700{ }^{\circ} \mathrm{C}$ interface, calculated with Eq. (1.9) is provided in the Table II.

From the Table II we can deduce that along with the interface characteristics (surface tension and contact angle), a great influence on the critical speed has the difference of density and the reinforcing particles size. Critical movement speed of the solidification front in order to incorporate the reinforcing particles has been calculated with Eq. (2.7) and is provided in the Table III.

The Table III shows us that for the same types of alloys and the same type of particles the movement critical speeds of the solidification front, plane and horizontal, is decisively influenced by the reinforcing particles sizes and by the difference between particle-solid, respectively particle-liquid inter-phase tensions.

For different systems, $\sigma_{\mathrm{PS}}$ and $\sigma_{\mathrm{PL}}$, respectively $\Delta \sigma_{0}$ values significantly differ, which leads to much bigger differences between the critical speeds calculated with Eq. (2.7).

The critical speed of the SiC particles at gas/liquid alloy interface.

\begin{tabular}{c|c|c|c|c|c|c|c}
\hline \hline no. & \multicolumn{2}{|c|}{ particles } & \multicolumn{4}{|c|}{ alloy } & contact \\
\cline { 2 - 6 } & type & $\begin{array}{c}\text { radius } \\
{\left[10^{-6} \mathrm{~m}\right]}\end{array}$ & mark & $\begin{array}{c}\text { density } \\
{\left[\mathrm{kg} / \mathrm{m}^{3}\right]}\end{array}$ & $\begin{array}{c}\text { surface } \\
\text { tension } \\
{\left[10^{3} \mathrm{~N} / \mathrm{m}\right]}\end{array}$ & & $\begin{array}{c}\left.{ }^{\circ}\right] \\
\text { speed } \\
{[\mathrm{m} / \mathrm{s}]}\end{array}$ \\
\hline 1 & $\mathrm{SiC}$ & 20 & ENACAlSi5Cu3Mg & 2456 & 690 & 125 & 4.973 \\
2 & $\mathrm{SiC}$ & 60 & ENACAlSi5Cu3Mg & 2456 & 690 & 125 & 2.871 \\
3 & $\mathrm{SiC}$ & 20 & ENACAlSi7Cu2Mg & 2477 & 708 & 127 & 5.160 \\
4 & $\mathrm{SiC}$ & 60 & ENACAlSi7Cu2Mg & 2477 & 708 & 127 & 2.979 \\
5 & $\mathrm{SiC}$ & 20 & AlMgSi0.5 & 2382 & 732 & 128 & 5.307 \\
6 & $\mathrm{SiC}$ & 60 & AlMgSi0.5 & 2382 & 732 & 128 & 3.064
\end{tabular}


TABLE III

The critical speed of the solidification front.

\begin{tabular}{c|c|c|c|c|c|c|c}
\hline \hline No. & Alloy & \multicolumn{2}{|c|}{ Particles } & $\sigma_{\mathrm{PS}}$ & $\sigma_{\mathrm{PL}}$ & $\Delta \sigma_{\mathrm{PS}}$ & $V_{c r}$ \\
& & Type & Radius, $\left[10^{-6} \mathrm{~m}\right]$ & {$[\mathrm{N} / \mathrm{m}]$} & {$[\mathrm{N} / \mathrm{m}]$} & {$[\mathrm{N} / \mathrm{m}]$} & {$\left[10^{-4} \mathrm{~m} / \mathrm{s}\right]$} \\
\hline 1 & ENACAlSi5Cu3Mg & $\mathrm{SiC}$ & 20 & 7.817 & 2.237 & 5.580 & 15.18 \\
2 & ENACAlSi5Cu3Mg & $\mathrm{SiC}$ & 60 & 7.817 & 2.237 & 5.580 & 1.68 \\
3 & ENACAlSi7Cu2Mg & $\mathrm{SiC}$ & 20 & 7.817 & 2.268 & 5.549 & 15.11 \\
4 & ENACAlSi7Cu2Mg & $\mathrm{SiC}$ & 60 & 7.817 & 2.268 & 5.549 & 1.51 \\
5 & AlMgSi0.5 & $\mathrm{SiC}$ & 20 & 7.817 & 2.290 & 5.527 & 14.95 \\
6 & AlMgSi0.5 & $\mathrm{SiC}$ & 60 & 7.817 & 2.290 & 5.527 & 2.43
\end{tabular}

\section{Experimental research on the characteristics of the composite materials reinforced with $\mathrm{SiC}$ particles}

The planning of the experiments has been realised according to Taguchi methodology [10]. Figure 4 presents, for the ENACAlSi5Cu3Mg alloy, the characteristic curve of traction test, and Fig. 5 presents the characteristic curve of traction test for the composite material sample that was obtained at the first experimental test. As shown in Fig. 5, the tensile strength for the obtained composite material has doubled.

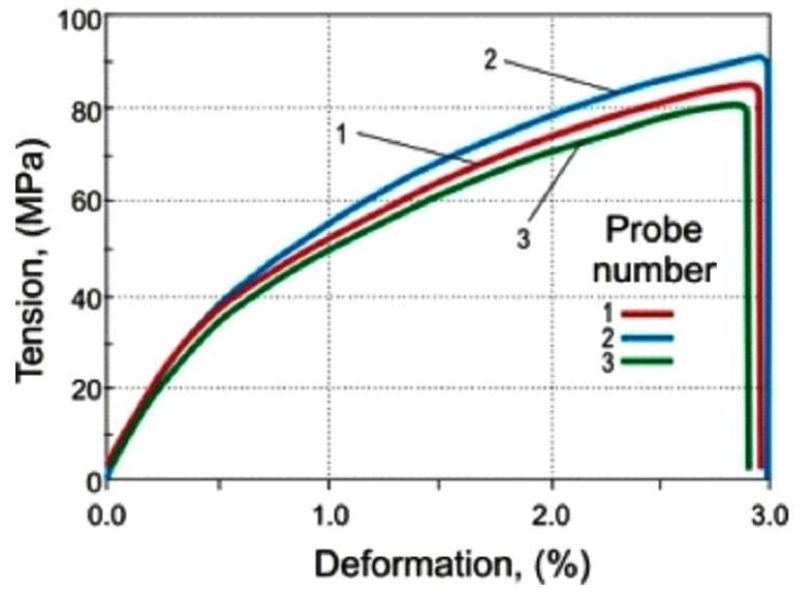

Fig. 4. Characteristic curve for base alloy.

The structure in Fig. 6a is characterized by: microporosity, average sized dendritic structure with uniform dendritic branches, a network of inter-metallic compounds, a network of inter-metallic compounds, a crowded area with potential rifts around the compounds, small Chinese letters. The structure in Fig. 6b is characterized by: potential cold drops, fine dendritic structure, with large grains, a network of inter-metallic compounds placed in inter-dendritic space and a small sized network of inter-metallic compounds. In the structure from Fig. 7a the existence of two components can be observed:

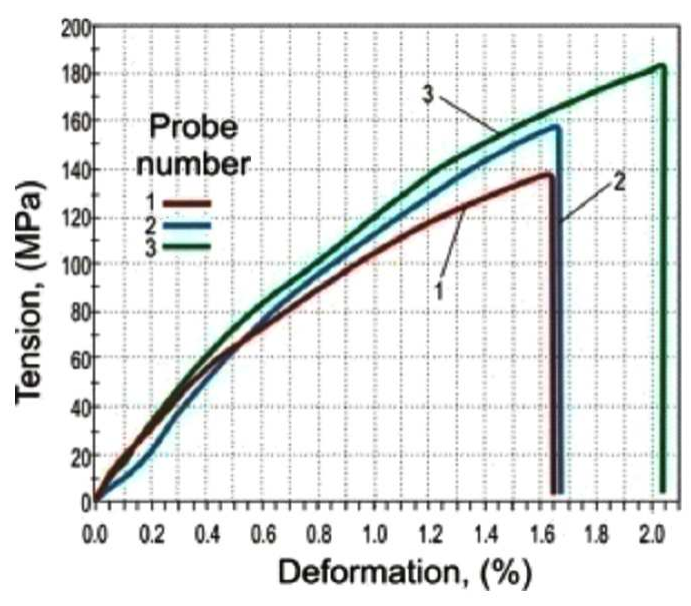

Fig. 5. Characteristic of curves or test number 1.
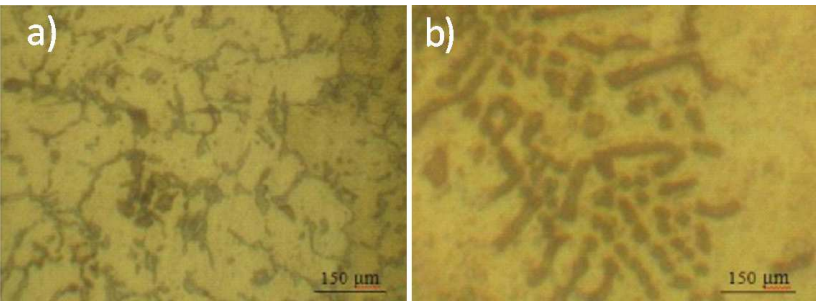

Fig. 6. The microphotos of matrix alloys:a - alloy ENACAlSi5Cu3, b - alloy ENACAlSi7Cu2Mg150.

aluminium alloy and $\mathrm{SiC}$ reinforcing particles having the $20 \mu \mathrm{m}$ dimensions. For the bigger particles (Fig. $7 \mathrm{~b}$ with a size of the particles of $60 \mu \mathrm{m}$ ) some of the causes of the fragmentation during the fragmentation process are likely to be seen (relatively high temperature, a strong shaking of the mixture of alloy/SiC particles).

In Fig. 8 is presented the image of a surface breaking obtained by SEM and EDX analysis of the break surface following attempt to traction of the composite ENACAlSi7Cu $\mathrm{Cu}_{2} \mathrm{Mg} / \mathrm{SiC}$ particles $(20 \mu \mathrm{m}$ and $60 \mu \mathrm{m})$. Note the brittle break of the composite and the exis- 


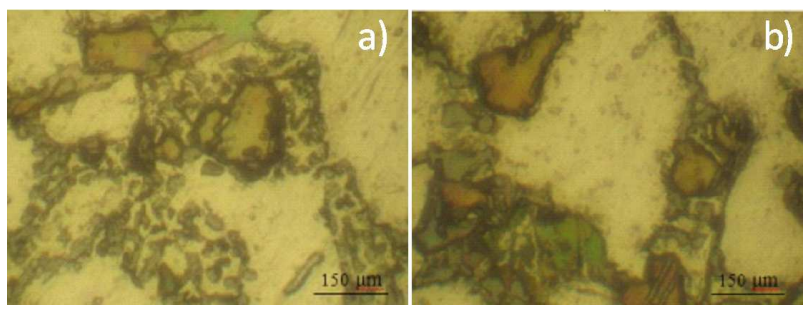

Fig. 7. The microphotos of obtained composites: a alloy ENACAlSi5Cu3/particles $\mathrm{SiC} 20 \mu \mathrm{m}$; b - alloy ENACAlSi7Cu2Mg/particles $\mathrm{SiC} 60 \mu \mathrm{m}$.
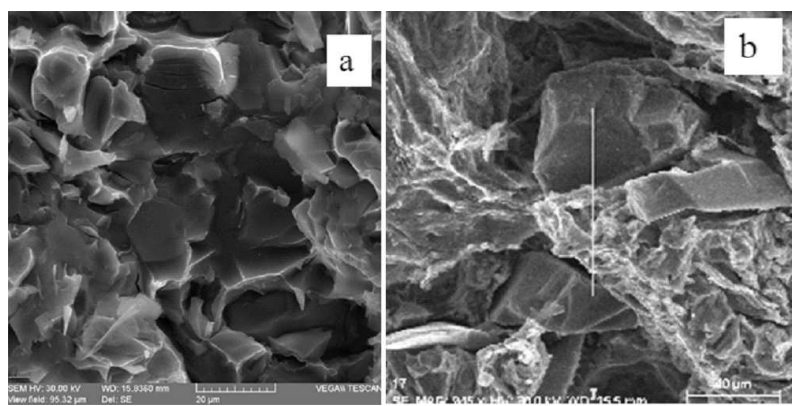

Fig. 8. The break imagines surface for ENACAlSi7Cu2Mg /particle $\mathrm{SiC}$ surface: a fractured surface imagine $(20 \mu \mathrm{m}) ; \quad$ b - fractured surface imagine $(60 \mu \mathrm{m})$.

tence of $\mathrm{SiC}$ particles in the fracture zone. For the ENACAlSi5Cu3Mg used alloy the variation of si and $\mathrm{C}$ distribution is presented in Fig. 9. The presence of the carbon in the Silicon Carbide particles is obvious and corresponds to a 30 percentage. At the, matrix level, a weak distribution of carbon can be observed, which confirms that during the processing the $\mathrm{Al}_{4} \mathrm{C}_{3}$ carbide has been formed, which improves the wetting process.

For realizing the ultrasonic test, a contact ultrasonic transducer has been used, with perpendicular beams or in delay line and a USLT 2000 (Krautkramer, Germany) control device. From the diagram control we can observe the presence of some very small sized discontinuities (porosities) which don't have a major influence on the subjected tensile specimens. From this reason, a very clear display of the end echo can be observed.

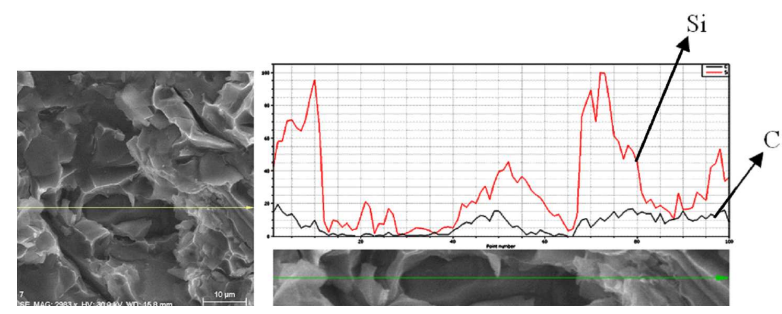

Fig. 9. The $\mathrm{Si}$ and $\mathrm{C}$ distribution along the reference line for the test number 1 .

\section{Conclusion}

The particles wetted by the liquid metal and with close density will be embedded in the melt without external energy input, and that dry and light need additional energy consumption (powder injection, melt stirring etc). The critical speed of $\mathrm{SiC}$ particles at the gas/liquid interface is influenced by the difference of density and reinforcing particles sizes. The critical movement speed of solidification front is decisively influenced by the difference between the inter-phase tensions $\sigma_{\mathrm{PS}}-\sigma_{\mathrm{PL}}$, as well as by reinforcing particles sizes, if their density significantly differs from the one of the liquid alloy. Pull strength doubled for some obtained composite material samples in the conditions of usage of a $2 \%$ and $6 \% \mathrm{SiC}$ particles mass percentage. Ultrasonic test of the obtained samples spotlighted the presence of some discontinuities of very small sizes (porosities) which don't have a major influence on the quality of the obtained composite material. The uniformity of the casting process is justified by the existence of the recorded end echo. The different sizes of the reinforcing particles indicate that during the mixing process the fragmentation of some particles after some cleavage plane has produced. From the analysis of the obtained structures one can see that in the central areas the aluminium has maximum concentration, and towards the separation limit between matrix and reinforcement one can find the presence of carbon. It is obviously that at the interface the aluminium carbide has formed, which leads to the improvement of the wetting process.

\section{References}

[1] P.K. Rohatgi, R. Asthana, Cast Reinforced Metal Composites, Eds. S.G. Fishman, A.K. Dhingra, World materials Congress, Chicago 1998, p. 61.

[2] A.S. Kacar, F. Rana, D.M. Stefanescu, Mat. Sci. Eng. A 1 35, (1991).

[3] B. Toshev, D. Platikanov, Colloids Surfaces A: Physicochemistry Engineering Aspects 291, 177 (2006).

[4] I. Carcea, in: Composite materials, Politehnium Publishing House, Iasi 2008 (in Romanian).

[5] D. Nedelcu, I. Carcea, G. Neagu, R. Zagan, L. Tabacaru, C. Predescu, in: Technologies for obtaining the composite materials, Politehnium Publishing House, Iasi 2009 (in Romanian).

[6] A. Ureńa, E. Martínez, E. Rodrigo, P. Gil, Comp. Sc. Techn. 64, 1843 (2004).

[7] G. Neagu, Fl. Ştefănescu, Rev. Metalurgia Sci, 11, 15 (2007).

[8] L.S. Gomez, K. Marinkovic, J.M. Torralba, M.E. Rabanal, O. Milosevic, Int. J. Modern Manufacturing Technologies I, 43 (2009).

[9] I. Carcea, M. Agop, Metal. Trans. 26, 3021 (1995).

[10] D. Nedelcu, O. Pruteanu, in: Aspects of external grooves obtaining by cold plastic deformation using Taguchi method, Tehnica-Info Publishing House, Chisinau 2000 (in Romanian). 